\title{
Differentiating Basal Insulin Preparations: Understanding How They Work Explains Why They Are Different
}

\author{
Alice Y. Y. Cheng (1) · Dhiren K. Patel · Timothy S. Reid $\cdot$ Kathleen Wyne
}

Received: December 10, 2018 / Published online: March 30, 2019

(c) The Author(s) 2019

\begin{abstract}
Since the introduction of insulin as a life-saving agent for patients with type 1 diabetes, insulin preparations have evolved to approximate physiologic insulin delivery profiles to meet prandial and basal insulin needs. While prandial insulins are designed to have quick time-action profiles that minimize postprandial glucose excursions, basal insulins are designed to have a protracted time-action profile to facilitate basal glucose control over $24 \mathrm{~h}$. Given that all insulins have the same mechanism of action at the target tissue level, the differences in time-action profiles are achieved through different mechanisms of protraction, resulting in different behaviors in the
\end{abstract}

Enhanced Digital Features To view enhanced digital features for this article go to https://doi.org/10.6084/ m9.figshare.7785305.

\footnotetext{
A. Y. Y. Cheng ( $\square)$

Department of Endocrinology and Metabolism, St

Michael's Hospital, 30 Bond St, Toronto,

ON M5B 1W8, Canada

e-mail: chenga@smh.ca

D. K. Patel

School of Pharmacy, MCPHS University, Boston,

MA, USA

T. S. Reid

Mercy Diabetes Center, Janesville, WI, USA

K. Wyne

The Ohio State University Wexner Medical Center, Columbus, $\mathrm{OH}$, USA
}

subcutaneous space and different rates of absorption into the circulation. Herein, we evaluate the differences in basal insulin preparations based on their differential mechanisms of protraction, and the resulting clinical action profiles. Multiple randomized control trials and real-world evidence studies have demonstrated that the newer second-generation basal insulin analogs, insulin glargine 300 units $/ \mathrm{mL}$ and insulin degludec 100 or 200 units $/ \mathrm{mL}$, provide stable glycemic control with once-daily dosing and are associated with a reduced risk of hypoglycemia compared with previous-generation basal insulin analogs insulin glargine 100 units/mL and insulin detemir. These advantages can lead to decreased healthcare resource utilization and cost. With this collective knowledge, healthcare providers and payers can make educated and well-informed decisions when determining which treatment regimen best meets the needs of each individual patient.

Funding: Sanofi US, Inc.

Keywords: Basal insulin; Hypoglycemia; Pharmacodynamics; Pharmacokinetics; Protraction; Second-generation long-acting insulin

\section{INTRODUCTION}

Since the introduction of insulin as a life-saving drug for patients with type 1 diabetes (T1D) in 
1922, insulin preparations have evolved to develop improved prandial and basal insulins to mimic pancreatic insulin secretion profiles. Under normal physiologic conditions, the secretion of insulin by pancreatic $\beta$-cells is a dynamic process that quickly responds to the changing needs of glycemic control of the individual throughout the day and night. In addition to maintaining fairly constant basal levels of insulin secretion during periods of fasting, the pancreas must also be capable of storing and releasing a surge of insulin to prevent rapid postprandial peaks in blood glucose levels. To artificially mimic this sophisticated pattern of insulin production and secretion, different formulations of exogenous insulin with complementary time-action profiles are required.

The development of basal insulins has been achieved by employing different means of protraction to prolong the rate of insulin absorption from the subcutaneous injection site into the circulation. From its origins as a crude extract of the pancreas of animals, insulin development has had a steady trajectory (Fig. 1), beginning with the addition of protamine to provide the first slow-release insulin in 1946 (neutral protamine Hagedorn [NPH] insulins) to the current inception of basal insulin analogs. Each advance has resulted in basal insulins with flatter pharmacokinetic (PK) and pharmacodynamic (PD) profiles, and longer durations of action. Given the many options to choose from, a better understanding of how the absorption of basal insulin is prolonged can be helpful in making the appropriate selection. Herein, we contrast the insulin preparations on the basis of their differential mechanisms of protraction and clinical impact. This article does not contain any studies with human participants or animals performed by any of the authors.

\section{BASAL INSULINS: MECHANISMS OF PROTRACTION}

Once absorbed into the circulation, all insulins have the same mechanism of action on target tissues (i.e., all insulins have the same action if administered intravenously). Therefore, the different time-action profiles of various insulins result from differences in their behavior in the subcutaneous space, which then affects the rate of absorption into the circulation (insulin protraction; Table 1). Protraction can be achieved using various methods, including the use of recombinant technology to modify the amino acid sequence; modifications to the formulation to influence self-association pre or post injection; the attachment of fatty acid linkers to allow reversible binding to serum albumin; and, more recently, by increasing the concentration delivered in combination with in vivo precipitation at the injection depot following administration to the individual.

\section{HUMAN BASAL INSULIN: NPH INSULIN}

NPH insulin, introduced in the 1940s, was one of the first intermediate-acting insulin formulations [1]. The addition of protamine results in a protamine-insulin complex that precipitates in solution before injection. After injection, the protamine-insulin complex diffuses slowly from the subcutaneous space, resulting in an intermediate-acting time-action profile [2].

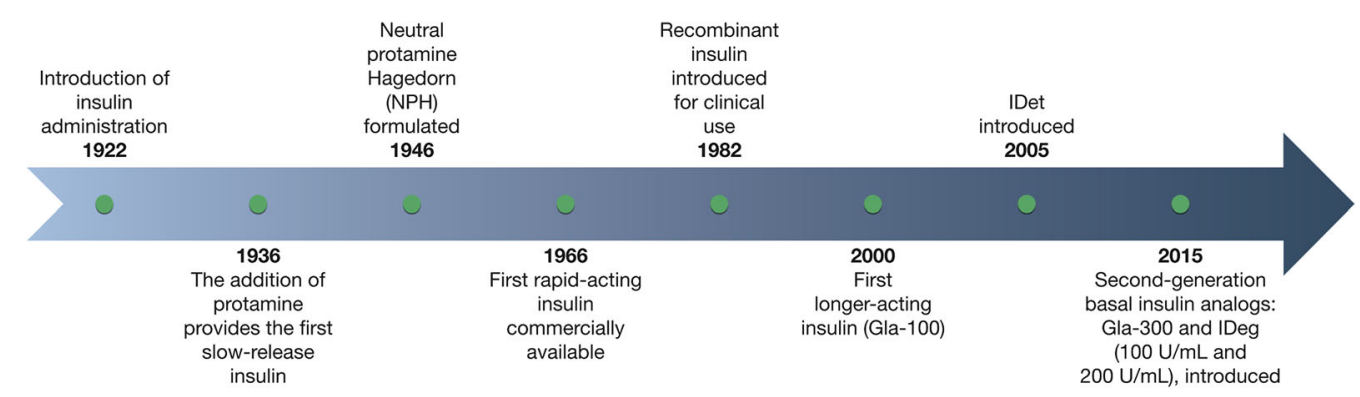

Fig. 1 Advances in insulin development. Gla-100 insulin glargine 100 units $/ \mathrm{mL}$, Gla-300 insulin glargine 300 units $/ \mathrm{mL}$, IDeg insulin degludec, IDet insulin detemir 
Table 1 Mechanisms of protraction of human regular insulin, NPH insulin, and first- and second-generation long-acting insulin analogs. Based on Heise and Mathieu [2]; Pandyarajan and Weiss [3]

\begin{tabular}{|c|c|c|}
\hline Insulin & Modification & Mechanism of protraction \\
\hline $\begin{array}{l}\text { Short-acting } \\
\text { regular insulin }\end{array}$ & $\begin{array}{l}\text { Nil (animal and } \\
\text { recombinant human } \\
\text { forms) }\end{array}$ & Nil \\
\hline $\begin{array}{l}\text { Intermediate- } \\
\text { acting NPH } \\
\text { insulin }\end{array}$ & $\begin{array}{l}\text { Nil (animal and } \\
\text { recombinant human } \\
\text { forms) }\end{array}$ & $\begin{array}{l}\text { Preformed precipitate of protamine-insulin conglomerates the crystals } \\
\text { of which are retained in "heaps" at injection depot }\end{array}$ \\
\hline Lispro & $\begin{array}{l}\text { Pro }^{\mathrm{B} 28} \rightarrow \text { Lys } \\
\text { Lys }^{\mathrm{B} 29} \rightarrow \text { Pro }\end{array}$ & More rapid circulation/action than regular human insulin \\
\hline Aspart & Pro $^{\mathrm{B} 28} \rightarrow$ Asp & More rapid circulation/action than regular human insulin \\
\hline Glulisine & $\begin{array}{l}\text { Asn }^{\mathrm{B} 3} \rightarrow \text { Lys } \\
\text { Lys }^{\mathrm{B} 29} \rightarrow \text { Glu }\end{array}$ & More rapid circulation/action than regular human insulin \\
\hline Gla-100 & $\begin{array}{l}\operatorname{Arg}^{\mathrm{B} 31}-\operatorname{Arg}^{\mathrm{B} 32} \operatorname{tag} \\
\operatorname{Asp}^{\mathrm{A} 21} \rightarrow \text { Gly }\end{array}$ & $\begin{array}{l}\text { Soluble in acidic } \mathrm{pH} \text { pre-injection. Forms microprecipitates while } \\
\text { equilibrating with physiologic } \mathrm{pH} \text { at the injection site; free glargine } \\
\text { then dissociates from the injection depot and is absorbed into the } \\
\text { circulation }\end{array}$ \\
\hline IDet & $\begin{array}{l}\text { Modification of Lys }{ }^{\mathrm{B} 29} \text { by a } \\
\text { tethered fatty acid }\end{array}$ & $\begin{array}{l}\text { Self-association at the injection depot as dihexamers and reversible } \\
\text { binding, via fatty acid linker, to albumin at the injection depot and in } \\
\text { the circulation }\end{array}$ \\
\hline Gla-300 & $\begin{array}{l}\operatorname{Arg}^{\mathrm{B} 31}-\operatorname{Arg}^{\mathrm{B} 32} \text { tag } \\
\operatorname{Asp}^{\mathrm{A} 21} \rightarrow \text { Gly }\end{array}$ & $\begin{array}{l}\text { Soluble in acidic } \mathrm{pH} \text { pre-injection. Precipitates at physiologic } \mathrm{pH} \text {, but } \\
\text { with more compact microprecipitates compared with Gla-100, } \\
\text { resulting in a reduced surface area from which more protracted } \\
\text { absorption can occur }\end{array}$ \\
\hline IDeg & $\begin{array}{l}\text { Modification of } \text { Lys }^{\mathrm{B} 29} \text { by a } \\
\text { dicarboxylic acid } \\
\text { Addition of a fatty acid side } \\
\text { chain }\end{array}$ & $\begin{array}{l}\text { Multihexamer chain formation at the injection depot, with dissociation } \\
\text { of zinc allowing hexamer breakdown as well as binding to serum } \\
\text { albumin via attached fatty acid linker }\end{array}$ \\
\hline
\end{tabular}

Gla-100 insulin glargine 100 units/mL, Gla-300 insulin glargine 300 units/mL, IDeg insulin degludec, IDet insulin detemir, NPH neutral protamine Hagedorn

\section{FIRST-GENERATION BASAL INSULIN ANALOGS: INSULIN GLARGINE 100 UNITS/ML AND INSULIN DETEMIR}

The first-generation basal insulin analogs were developed using recombinant deoxyribonucleic acid technology to alter the amino acid sequence of human insulin to create the desired effects. The first of these basal insulin analogs, insulin glargine 100 units/mL (Gla-100) became available in the year 2000 and was soon followed by insulin detemir (IDet) in 2005 [1]. In the case of Gla-100, the A21-asparagine of human insulin is replaced by glycine, and two arginine residues are added to $\mathrm{B} 30$. These modifications increase the isoelectric point of the insulin analog, making it soluble in acidic conditions (before injection), but when exposed 
to the higher (physiologic) $\mathrm{pH}$ of the human subcutaneous space after injection, microprecipitates form in the injection depot $[3,4]$. Monomeric Gla-100 is then slowly released into the circulation from the microprecipitate of the injection depot (a process that depends on the surface area of the injection depot), thereby protracting the PK profile of insulin glargine [2]. In the case of IDet, the amino acid sequence of the human insulin is altered and a 14-carbon myristoyl fatty acid is added to the B29-lysine residue. This results in a soluble insulin that self-associates into dihexamers in the subcutaneous space and reversibly binds to albumin via the fatty acid linker, allowing for slower release of monomeric free insulin [2].

\section{SECOND-GENERATION BASAL INSULIN ANALOGS: INSULIN GLARGINE 300 UNITS/ML AND INSULIN DEGLUDEC 100 UNITS/ML AND 200 UNITS/ML}

Although the first-generation basal insulin analogs represented a significant advance in basal insulin technology, allowing for less hypoglycemia compared with NPH insulin, there was still residual hypoglycemia risk, and further improvements in the time-action profile were needed. The second-generation basal insulin analogs insulin glargine 300 units $/ \mathrm{mL}$ (Gla-300) and insulin degludec (IDeg) were introduced in 2015.

Gla-300 is a different formulation of glargine, whereby the insulin is delivered in a smaller (one-third) volume of liquid. Interestingly, this results in an insulin with an altered PK profile, with even longer and flatter effects than Gla-100. Given that Gla-100 and Gla-300 are both insulin glargine, how can the time-action profiles be different? To answer this, one must consider the unique mechanism by which glargine protracts its time in the subcutaneous space. Gla-300 delivers the same dosage of insulin as Gla-100, but in one-third of the volume. This results in reduced surface area of injection depot, ultimately resulting in a slower and more gradual release of monomers of Gla-300 as compared with Gla-100 (Fig. 2) [4].

IDeg utilizes a different method of protraction (Fig. 3). Like IDet, it also has a fatty acid

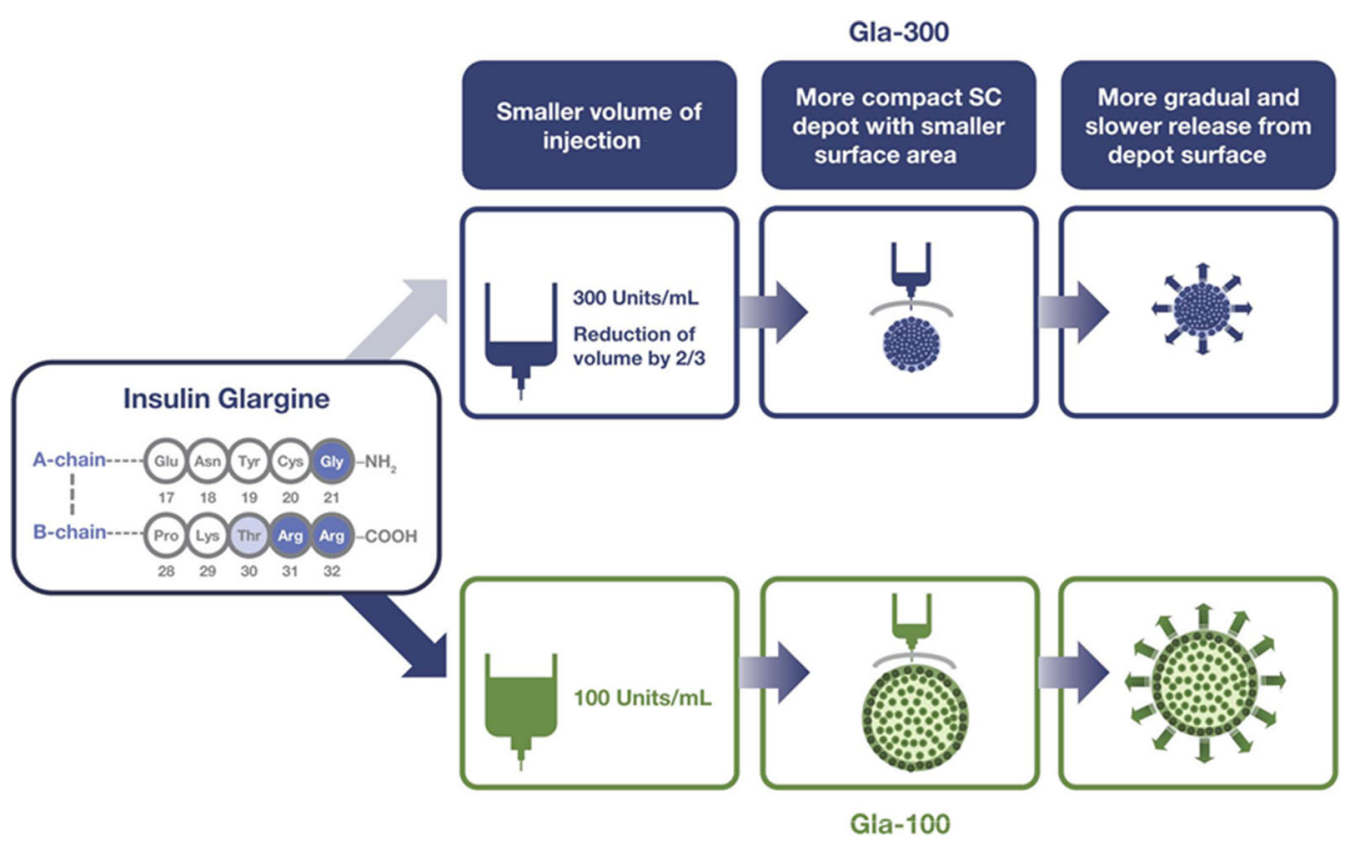

Fig. 2 Compact depot formation with Gla-300 results in more gradual insulin release as compared with Gla-100 $[1,27,48,49]$. Gla-100 insulin glargine 100 units $/ \mathrm{mL}$, Gla-300 insulin glargine $300 \mathrm{units} / \mathrm{mL}, S C$ subcutaneous 
side chain that allows for albumin binding. In addition, it exists as dihexamers in solution, and upon injection into the subcutaneous space, the removal of the phenol component allows for self-association into multihexamer chains. Over time, the zinc in IDeg dissociates and the hexamers are released and then dissociate into monomers that enter the circulation [2]. IDeg is available as 100 units $/ \mathrm{mL}$ or 200 units $/ \mathrm{mL}$, but, in the case of these preparations, the time-action profile is not impacted by the change in concentration.

The act of concentrating insulin and reducing injection volume does not automatically alter the time-action profile. An understanding of the mechanism of protraction is important to explain this. In the case of Gla-300, the mechanism of protraction is through microprecipitation in the subcutaneous space. Reducing the injection volume reduces the surface area of the microprecipitates and slows dissolution and absorption [2]. However, in the case of IDeg, reducing volume does not affect the multihexamer formation or its protraction in the subcutaneous space. Therefore, IDeg 200 units/mL (IDeg-200) behaves the same as IDeg 100 units/ $\mathrm{mL}$ (IDeg-100) [5].

\section{FROM PHYSICAL PROPERTIES TO PK/PD PROFILE}

The mechanism of protraction has a significant impact on the PK and PD properties. When assessing these parameters, serum insulin concentration-versus-time and glucose infusion rate (GIR) are key measures. The euglycemic glucose clamp procedure is the gold standard for measuring total body glucose disposal in response to the administered test insulin to determine the PK/PD characteristics of the compound [6]. The GIR curve depicts the glucose-metabolizing ability of any given insulin preparation over time, with the peak GIR

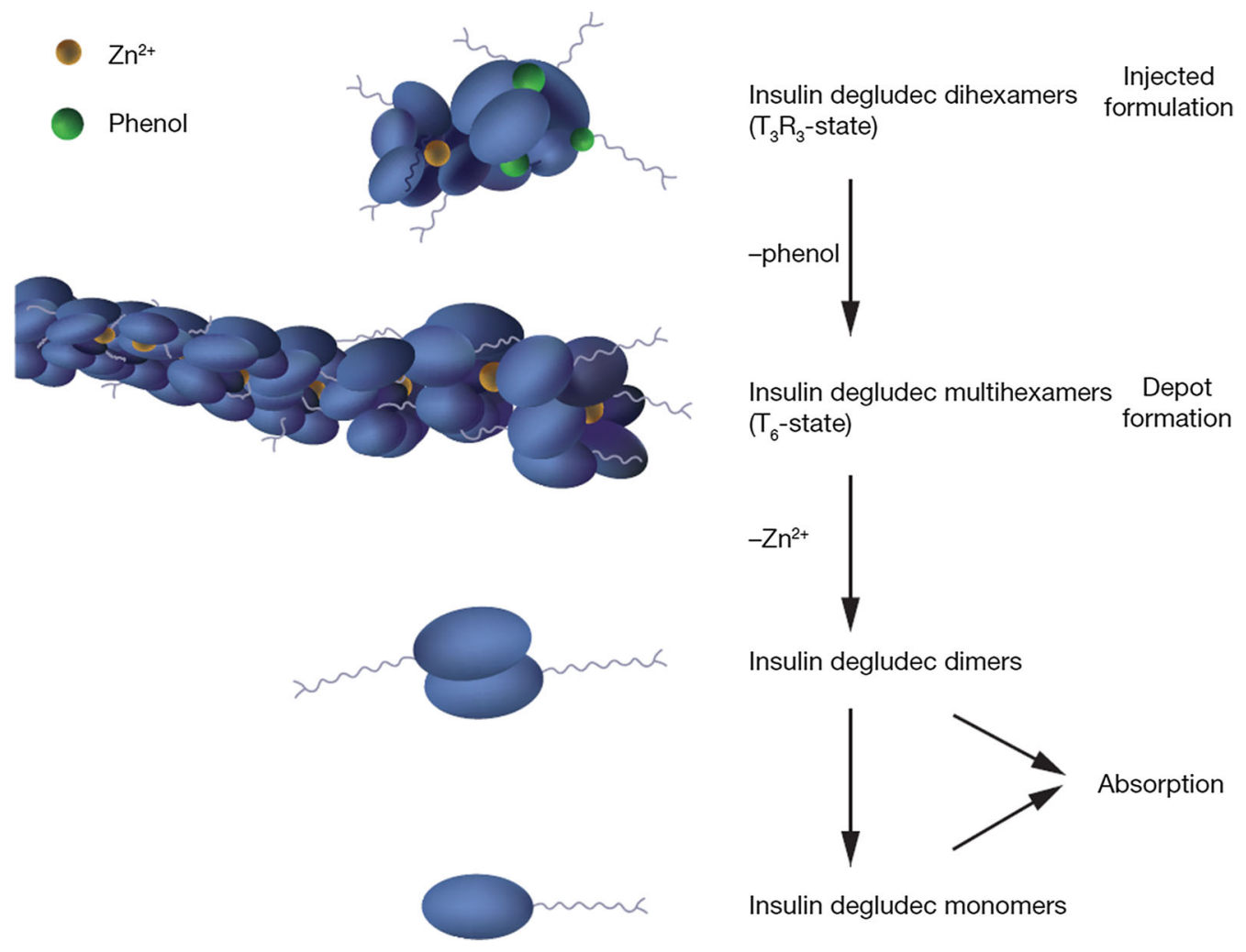

Fig. 3 Mode of protraction of IDeg. IDeg insulin degludec, $Z n$ zinc. Reproduced from Jonassen et al. [50] 


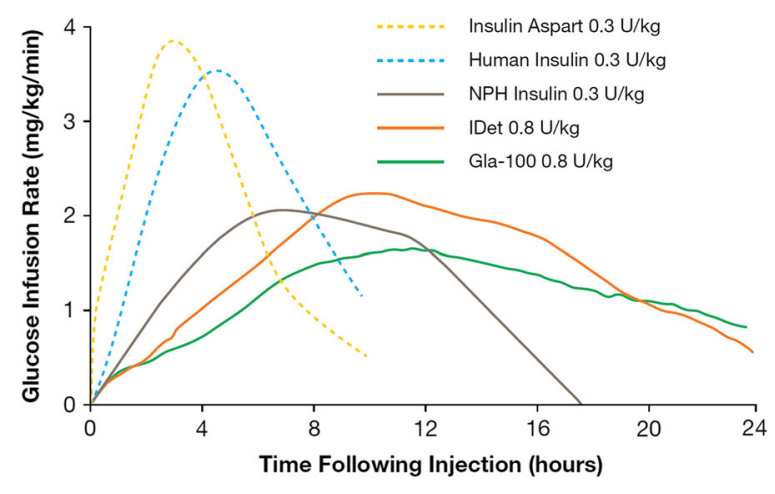

Fig. 4 Glucose-lowering effect of different insulin preparations based on data from published PD studies of patients with T2D. Gla-100 insulin glargine 100 units/ $\mathrm{mL}$, IDet insulin detemir, NPH neutral protamine Hagedorn, $P D$ pharmacodynamic, T2D type 2 diabetes. Adapted from Evans, 2011 [7] (C) 2011, Blackwell Publishing Ltd.

occurring when higher levels of glucose need to be administered during the euglycemic clamp to balance the effect of the administered insulin and maintain euglycemia [6].

When compared with regular insulin and rapid-acting insulin analogs, such as insulin aspart, the pre-injection precipitation associated with NPH insulin achieves a flatter and longer PD profile. However, it is not sufficient to cover the entire 24-h period (Fig. 4). Post-injection micro-precipitation (Gla-100) or dimerization and albumin binding (IDet) produce more gradually distributed and prolonged PD profiles that more closely mimic normal physiologic basal insulin levels and glucodynamic effects (Fig. 4) $[2,3,6$, 7]. Further prolongation of the PK and PD profiles has now been achieved with second-generation basal insulin analogs, such as Gla-300 and IDeg [8-10] (Table 1; Figs. 5, 6).

The longer time-action profiles of the second-generation basal insulin analogs require consideration of two important clinical factors-dose splitting and insulin stacking. Splitting basal insulin doses into twice-daily administration is required when the basal insulin does not provide a prolonged glucoselowering effect over a period of $24 \mathrm{~h}$. Higher doses of Gla-100, which is usually administered once daily, have been associated with longer durations of action ( $>24 \mathrm{~h}$ ) [11]. The second- generation basal insulin analogs have longer mean durations of action (approximately $32 \mathrm{~h}$ for Gla-300 and at least $42 \mathrm{~h}$ for IDeg), and therefore do not require dose splitting [2], which provides a convenience advantage to the patient. With these newer formulations, a oncedaily dose can be administered using a pen device that can deliver up to 160 units of Gla300 or IDeg-200. This larger delivery dose reduces the number of injections and the number of pens required.

The concept of insulin stacking is associated with prandial insulins with short half-lives. It refers to the unwanted accumulation of insulin following multiple injections of correctional doses of prandial insulin at close intervals and is associated with an increased risk of hypoglycemia [12, 13]. Concerns have been raised over the potential for insulin stacking with daily dosing of second-generation basal insulin
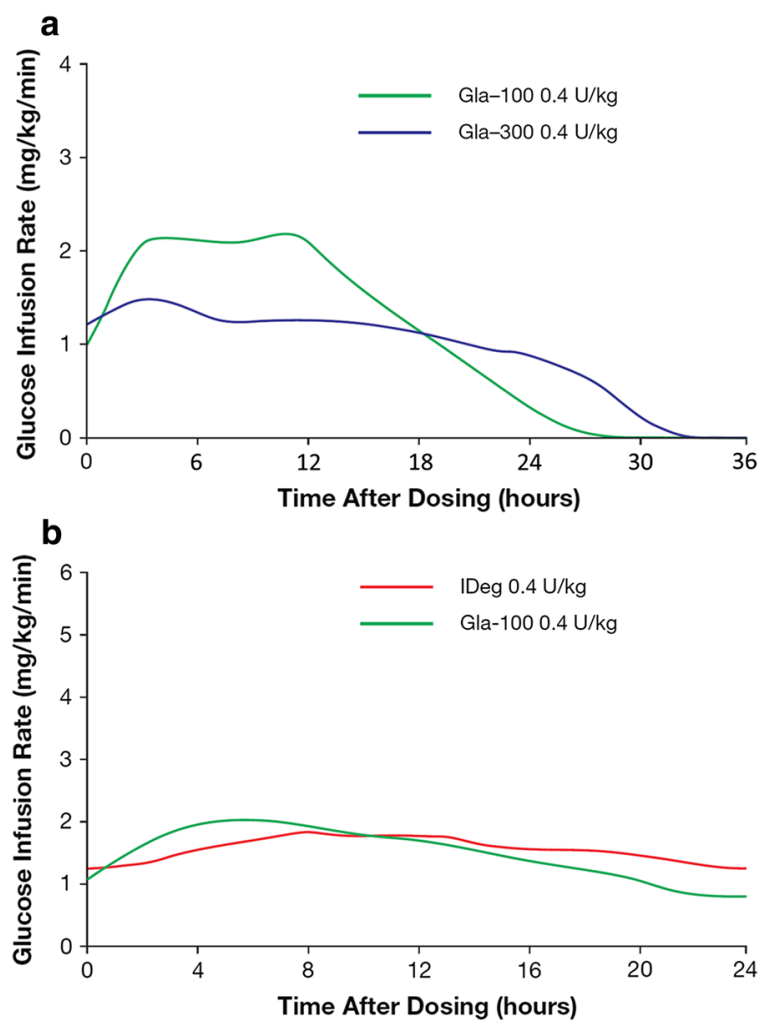

Fig. 5 Glucose infusion rate profile of a Gla-300 and b IDeg compared with Gla-100. Gla-100 insulin glargine 100 units/mL, Gla-300 insulin glargine 300 units $/ \mathrm{mL}$, IDeg insulin degludec. Reproduced with permission from Becker et al. [27] (C) 2015, American Diabetes Association 

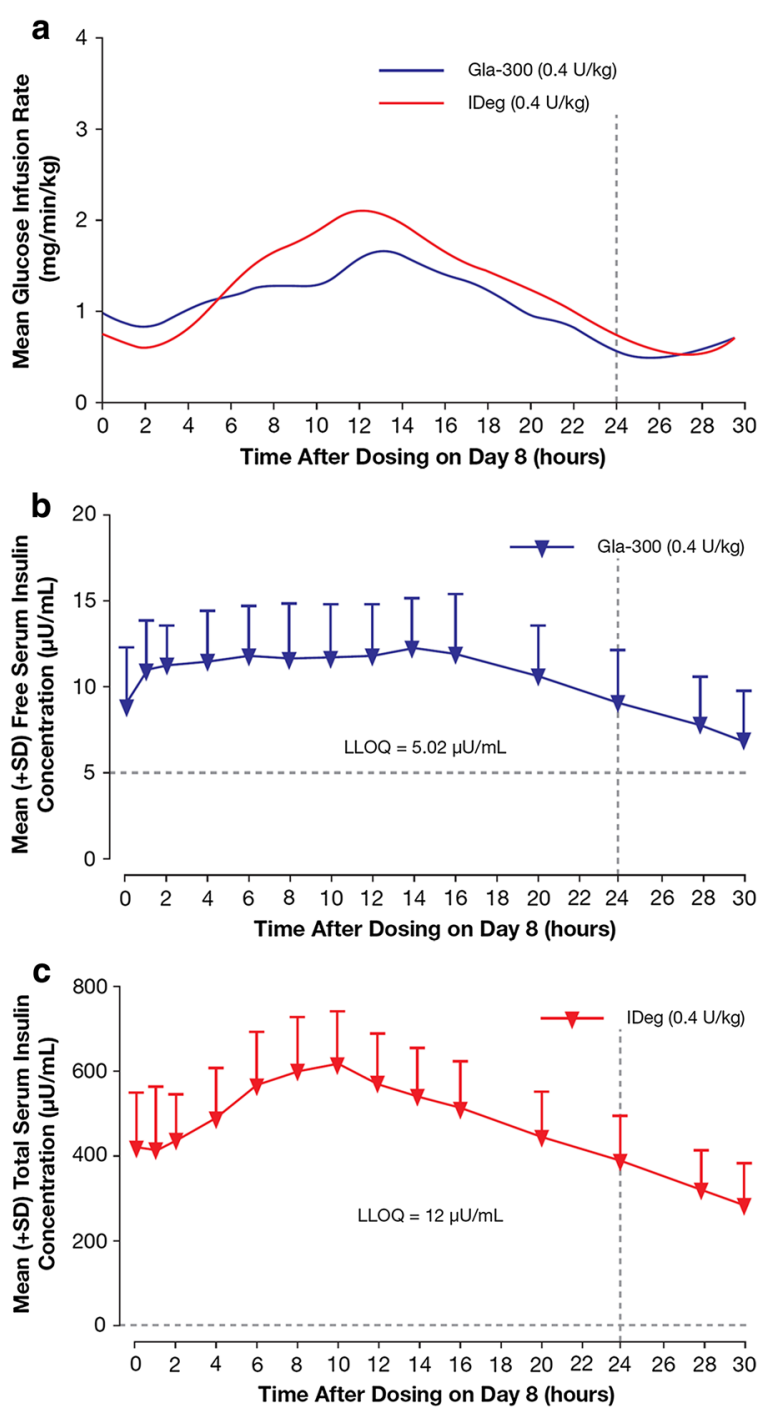

Fig. 6 a Mean glucose infusion rate profile, $\mathbf{b}$ mean free serum insulin concentration of Gla-300, and $\mathbf{c}$ mean total serum concentration of IDeg-100 in patients with T1D. Gla-300 insulin glargine 300 units $/ \mathrm{mL}$, IDeg insulin degludec, $L L O Q$ lower limit of quantification, $S D$ standard deviation. Reproduced from Bailey et al. [10] (C) 2017, The Authors

analogs with a duration of action longer than $24 \mathrm{~h}$. However, the distinction needs to be made between "stacking" and the appropriate accumulation of long-acting insulin preparations at steady state. With appropriate dosing and adjustment intervals, stacking is not an issue with long-acting basal insulins with a duration of action longer than $24 \mathrm{~h}$ [14-17]. A $1: 1$ dose conversion is recommended for patients already taking long- or intermediateacting basal insulins for both second-generation products, with a weight-based initial dose for insulin-naïve patients starting on Gla-300, and 10 units per day for those starting on IDeg. For both Gla-300 and IDeg, a 3- to 4-day gap between dose increases is recommended by the manufacturers, with a 2-unit decrease if fasting plasma glucose is below goal, 2-unit increase if above goal, and no change if within goal. Titration of both products should be individualized on the basis of the patient's needs and goals $[18,19]$. In randomized controlled trials, Gla-300 has proven efficacy and safety in a range of simple 2- to 4-step algorithms (Fig. 7) [20-26]. Healthcare providers may select the appropriate schedule based upon a patient's individual needs. Similarly, IDeg was demonstrated to be effective and well tolerated when either a simple or step-wise weekly titration schedule was used (Fig. 7) [20-26].

\section{FROM PK/PD PROFILE TO CLINICAL OUTCOMES}

\section{Second-Generation Basal Insulin Analogs Compared with First-Generation}

When compared with Gla-100, Gla-300 is associated with a flatter and more consistent glucose-lowering effect, with more evenly distributed PK/PD profiles (Fig. 5). When assessed in a euglycemic clamp study, Gla-300 provided a smoother profile over a 24 -h period when compared with Gla-100 (Fig. 5a) [27]. Patients receiving Gla-300 had a significantly longer time to $50 \%$ of the area under the serum insulin and GIR curves ( $36 \mathrm{~h}$ ) compared with those receiving Gla-100 ( $28 \mathrm{~h})$, with smaller changes in steady-state concentrations and GIR up to $36 \mathrm{~h}$. When assessed by continuous glucose monitoring, Gla-100 and Gla-300 had a similar time in range [28]. Although similar profiles were seen with Gla-300 injected in the morning or the evening, larger glycemic excursions were seen for Gla-100 injected in the morning [28]. From a clinical perspective, the EDITION program demonstrated that Gla-300 reduces the risk of hypoglycemia (severe and 


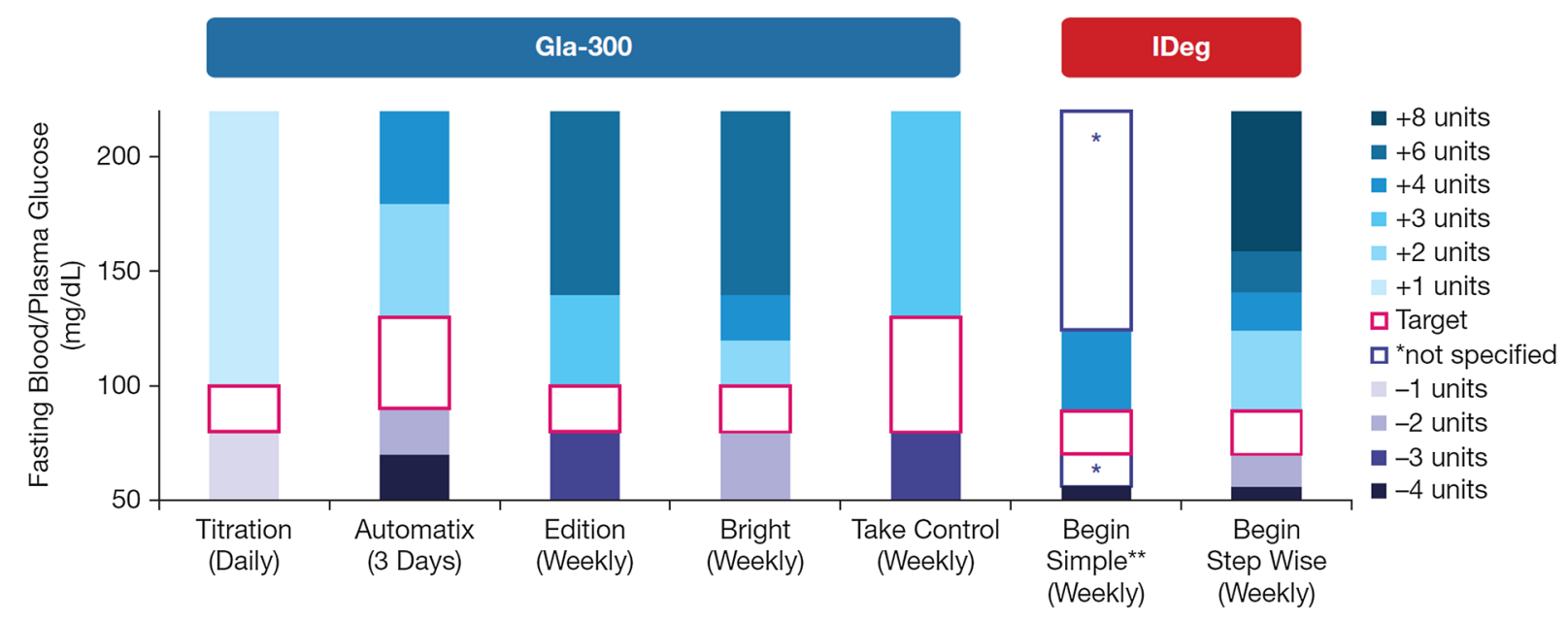

Fig. 7 Titration of Gla-300 and IDeg using different algorithms. *Not specified in algorithm; ${ }^{* *}$ Dose adjustment $>126 \mathrm{mg} / \mathrm{dL}$ not specified in algorithm; Gla-300 insulin glargine 300 units $/ \mathrm{mL}$, IDeg insulin degludec. Adapted

nocturnal confirmed), with similar glycemic control compared with Gla-100 in treat-to-target studies [20, 21, 29-32]. In most of the EDITION clinical trials, annualized rates of nocturnal confirmed $(<3.9 \mathrm{mmol} / \mathrm{L})$ or severe nocturnal hypoglycemia were lower with Gla300 than Gla-100, with risk ratios of $0.79,0.63$, 0.89 , and 0.62 for the EDITION 1, 2, 3, and JP1 trials, respectively. However, a non-significant difference in overall confirmed hypoglycemia rates was observed between Gla-300 and Gla100 in the EDITION 4 trial (0.98-1.16) for the study as a whole. A randomized controlled study in people with T1D using continuous glucose monitoring also found that nocturnal confirmed and severe hypoglycemia rates were lower with Gla-300 than with Gla-100 (4.0 versus 9.0 events per participant-year) [28].

Similar to Gla-300, IDeg provided much smoother profiles over $24 \mathrm{~h}$, with a considerably longer half-life than Gla-100 (Fig. 5b) [33]. Across all doses tested in a 42-h euglycemic clamp study, IDeg GIR profiles were flatter and more stable across the assessed 6-h intervals than those for Gla-100, with estimated halflives of $25.4 \mathrm{~h}$ and $12.1 \mathrm{~h}$, respectively [33]. The BEGIN program demonstrated that IDeg also reduces the risk of hypoglycemia, with similar glycemic control compared with Gla-100 in from Riddle et al. [20]; Bolli et al. [21]; Rosenstock et al. [22]; Yki-Järvinen et al. [23]; Davies et al. [24]; PhilisTsimikas et al. [25]; Strojek et al. [26]

treat-to-target studies [34]. Overall, there was a $21 \%$ lower rate of confirmed hypoglycemia (risk ratio 0.79 ) and a $52 \%$ lower rate of nocturnal confirmed hypoglycemia (risk ratio 0.48 ) with IDeg compared with Gla-100. In the SWITCH 1 and 2 clinical trials, people with T1D or type 2 diabetes (T2D) treated with IDeg had reduced rates of overall symptomatic hypoglycemia compared with those treated with Gla-100 (SWITCH 1, risk ratio 0.89; SWITCH 2, risk ratio $0.70)[35,36]$. In the DEVOTE study, participants with T2D receiving IDeg had significantly lower rates of severe hypoglycemia compared with those receiving Gla-100 (4.9\% vs. $6.6 \%$, respectively; rate ratio 0.60 ) [37].

Real-world studies of Gla-300 and IDeg have confirmed less hypoglycemia compared with Gla-100 in T2D [38-42]. In the DELIVER 2 and 3 studies, significantly fewer patients treated with Gla-300 experienced hypoglycemia compared with Gla-100 or IDet (adjusted odds ratio 0.75 and 0.43 , respectively). Similar hypoglycemia rates were seen in patients switching to Gla-300 and IDeg from other basal insulins in the DELIVER D+ study (adjusted odds ratio 0.97). Across the EDITION clinical trials program, a higher mean dose (10-20\%) of Gla-300 was required compared with Gla-100. This increase in dose is again explained by the mechanism of 
protraction. The microprecipitate of Gla-300, with its smaller surface area, remains in the subcutaneous space longer, presumably exposing it to tissue proteases, resulting in local degradation. As these higher dose needs are clinically relevant, they must be considered when switching from Gla-100 to Gla-300. Although randomized trials have demonstrated a higher insulin dose requirement of Gla-300, in real-world studies, the requirement is not consistent and may potentially be, at least partly, explained by differences in the treated populations [43]. Insulin dose requirements have been assessed in the ACHIEVE CONTROL pragmatic real-life study, which compared basal insulins in insulin-naïve patients with T2D [44]. Results show that, compared with other basal insulins used in standard of care at similar doses, patients treated with Gla-300 were more likely to achieve glycated hemoglobin targets without hypoglycemia [44].

\section{Second-Generation Basal Insulin Analogs Compared with Each Other}

The benefits of second-generation basal insulin analogs over first-generation are well established. Currently, comparison within class is clinically meaningful and such comparisons are emerging. In a study that compared steady-state PK and PD profiles of Gla-300 and IDeg-100 in patients with T1D, Gla-300 provided a steadier PD profile (20\% lower within-day fluctuation in GIR) and a more evenly distributed PK profile (Fig. 6) at a 0.4 units $/ \mathrm{kg} /$ day dose; however, no significant differences were seen at the higher 0.6 units $/ \mathrm{kg} /$ day dose [10]. A trial-level metaanalysis of the EDITION and BEGIN programs offered indirect comparison [45]. In the DELIVER D+ study, a direct comparison of realworld clinical outcomes with Gla-300 and IDeg showed that people with T2D switching from other basal insulins to two second-generation basal insulin analogs had comparable glycemic control, and incidence and rates of hypoglycemia [42]. Real-world analysis of electronic health records in the LIGHTNING study also demonstrated similar levels of glycemic control across basal insulins, but reported significantly lower rates of severe hypoglycemia in patients switching to Gla-300 or IDeg, compared with Gla-100 or IDet [46]. Among insulin-naïve patients with T2D, the CONFIRM real-world analysis showed that the group receiving IDeg had less hypoglycemia, greater glycemic control, and improved insulin retention compared with Gla-300 [47]. However, there are limitations to real-world observational analyses, with randomized controlled clinical trials needed to better understand differences between therapies. The BRIGHT study is the first head-to-head randomized controlled trial comparing Gla-300 with IDeg in insulin-naïve patients with T2D. The study demonstrated comparable levels of glycemic control with similar overall incidence and rates of hypoglycemia. However, hypoglycemia was lower with Gla-300 during the first half of the study (titration period) when the greatest glucose reduction and insulin dose increase occurred [22].

\section{CONCLUSIONS}

The time-action profile of an insulin preparation is determined by its time course and pattern of absorption and distribution from the subcutaneous injection site. Basal insulins have distinct mechanisms of protraction that give rise to equally distinct $\mathrm{PK} / \mathrm{PD}$ profiles and accompanying clinical properties in people with T1D and T2D. Understanding these differential mechanisms is important to explain the clinical benefits and differences of the second-generation basal insulin analogs Gla-300 and IDeg over the earlier generation of basal insulins, Gla-100 and IDet. Gla-300 and IDeg show longer duration of action and smoother PK/PD profiles than these earlier-generation basal insulin analogs, leading to smaller glycemic excursions and a lowered risk of hypoglycemia, while retaining similar levels of glycemic control. Understanding the differences among first- and second-generation basal insulin analogs aids healthcare providers in making the most appropriate treatment decisions to address individual patient needs. 


\section{ACKNOWLEDGEMENTS}

Funding. Development of the manuscript, article processing charges and the open access fee were funded by Sanofi US, Inc. All authors had full access to the articles reviewed in the manuscript and take complete responsibility for the integrity and accuracy of the manuscript.

Medical Writing and Editorial Assistance. The authors received writing/editorial support in the preparation of this manuscript provided by Rasilaben Vaghjiani, PhD, of Excerpta Medica, funded by Sanofi US, Inc.

Authorship. All named authors meet the International Committee of Medical Journal Editors (ICMJE) criteria for authorship for this article, take responsibility for the integrity of the work as a whole, and have given their approval for this version to be published. The sponsor also provided medical, regulatory, legal, and IP review of the final draft manuscript; suggestions were incorporated at the authors' discretion.

Disclosures. Alice Cheng has received honoraria for consulting or speaking for Abbott, Astra Zeneca, Boehringer Ingelheim, HLS Therapeutics, Janssen, Merck, Novo Nordisk, and Sanofi. She is also is in clinical trial involvement with Boehringer Ingelheim, Eli Lilly, and Sanofi. Dhiren Patel has been a speaker for AstraZeneca, Boehringer Ingelheim, Merck, Novo Nordisk, Sanofi, and Valeritas; and consulted for Becton and Dickinson, Eli Lilly, Merck, and Sanofi. Timothy Reid is a speaker/consultant for AstraZeneca, Intarcia, Janssen, Lilly, Novo Nordisk, and Sanofi-Aventis. Kathleen Wyne has consulted for AstraZeneca and Sanofi.

Compliance with Ethics Guidelines. This article does not contain any studies with human participants or animals performed by any of the authors.

Data Availability. Data sharing is not applicable to this article as no datasets were generated or analyzed during the current study.
Open Access. This article is distributed under the terms of the Creative Commons Attribution-NonCommercial 4.0 International License (http://creativecommons.org/licenses/ by-nc/4.0/), which permits any noncommercial use, distribution, and reproduction in any medium, provided you give appropriate credit to the original author(s) and the source, provide a link to the Creative Commons license, and indicate if changes were made.

\section{REFERENCES}

1. Pettus J, Santos Cavaiola T, Tamborlane WV, Edelman S. The past, present, and future of basal insulins. Diabetes Metab Res Rev. 2016;32:478-96.

2. Heise T, Mathieu C. Impact of the mode of protraction of basal insulin therapies on their pharmacokinetic and pharmacodynamic properties and resulting clinical outcomes. Diabetes Obes Metab. 2017;19:3-12.

3. Pandyarajan V, Weiss MA. Design of non-standard insulin analogs for the treatment of diabetes mellitus. Curr Diab Rep. 2012;12:697-704.

4. Anderson JE. An evolutionary perspective on basal insulin in diabetes treatment: innovations in insulin: insulin glargine U-300. J Fam Pract. 2016;65:S23-8.

5. Korsatko S, Deller S, Koehler G, et al. A comparison of the steady-state pharmacokinetic and pharmacodynamic profiles of 100 and $200 \mathrm{U} / \mathrm{mL}$ formulations of ultra-long-acting insulin degludec. Clin Drug Investig. 2013;33:515-21.

6. Arnolds S, Kuglin B, Kapitza C, Heise T. How pharmacokinetic and pharmacodynamic principles pave the way for optimal basal insulin therapy in type 2 diabetes. Int J Clin Pract. 2010;64:1415-24.

7. Evans M, Schumm-Draeger PM, Vora J, King AB. A review of modern insulin analogue pharmacokinetic and pharmacodynamic profiles in type 2 diabetes: improvements and limitations. Diabetes Obes Metab. 2011;13:677-84.

8. Heise T, Nosek L, Bøttcher SG, Hastrup H, Haahr H. Ultra-long-acting insulin degludec has a flat and stable glucose-lowering effect in type 2 diabetes. Diabetes Obes Metab. 2012;14:944-50.

9. Goldman J, Kapitza C, Pettus J, Heise T. Understanding how pharmacokinetic and 
pharmacodynamic differences of basal analog insulins influence clinical practice. Curr Med Res Opin. 2017;33:1821-31.

10. Bailey TS, Pettus J, Roussel R, et al. Morning administration of $0.4 \mathrm{U} / \mathrm{kg} /$ day insulin glargine $300 \mathrm{U} / \mathrm{mL}$ provides less fluctuating 24-hour pharmacodynamics and more even pharmacokinetic profiles compared with insulin degludec $100 \mathrm{U} / \mathrm{mL}$ in type 1 diabetes. Diabetes Metab. 2018;44:15-21.

11. Wang Z, Hedrington MS, Gogitidze Joy N, et al. Dose-response effects of insulin glargine in type 2 diabetes. Diabetes Care. 2010;33:1555-60.

12. Heise $\mathrm{T}$, Meneghini LF. Insulin stacking versus therapeutic accumulation: understanding the differences. Endocr Pract. 2014;20:75-83.

13. Walsh J, Roberts R, Heinemann L. Confusion regarding duration of insulin action: a potential source for major insulin dose errors by bolus calculators. J Diabetes Sci Technol. 2014;8:170-8.

14. Heise T, Bott S, Rave K, Dressler A, Rosskamp R, Heinemann L. No evidence for accumulation of insulin glargine (LANTUS): a multiple injection study in patients with type 1 diabetes. Diabet Med. 2002;19:490-5.

15. Bott $S$, Tusek C, Jacobsen LV, et al. Insulin detemir under steady-state conditions: no accumulation and constant metabolic effect over time with twice daily administration in subjects with type 1 diabetes. Diabet Med. 2006;23:522-8.

16. Aye MM, Atkin SL. Patient safety and minimizing risk with insulin administration-role of insulin degludec. Drug Healthc Patient Saf. 2014;6:55-67.

17. Danne T, Becker RH, Ping L, Philotheou A. Insulin glargine metabolite 21(A)-Gly-human insulin (M1) is the principal component circulating in the plasma of young children with type 1 diabetes: results from the PRESCHOOL study. Pediatr Diabetes. 2015;16:299-304.

18. Tresiba $^{\circledR}$ dosing and device. https://www. tresibapro.com/dosing-and-device/startingpatients.html. Accessed 23 Feb 2019.

19. Toujeo ${ }^{\circledR}$ insulin dosing and titration calculator. https://www.toujeopro.com/toujeo-insulin-dosingand-titration-calculator. Accessed 23 Feb 2019.

20. Riddle MC, Bolli GB, Ziemen M, et al. New insulin glargine 300 units/mL versus glargine 100 units $/ \mathrm{mL}$ in people with type 2 diabetes using basal and mealtime insulin: glucose control and hypoglycemia in a 6-month randomized controlled trial (EDITION 1). Diabetes Care. 2014;37:2755-62.
21. Bolli GB, Riddle MC, Bergenstal RM, et al. New insulin glargine $300 \mathrm{U} / \mathrm{ml}$ compared with glargine $100 \mathrm{U} / \mathrm{ml}$ in insulin-naïve people with type 2 diabetes on oral glucose-lowering drugs: a randomized controlled trial (EDITION 3). Diabetes Obes Metab. 2015;17:386-94.

22. Rosenstock J, Cheng A, Ritzel R, et al. More similarities than differences testing insulin glargine 300 units/mL versus insulin degludec 100 units $/ \mathrm{mL}$ in insuline-naïve type 2 diabetes: the randomized head-to-head BRIGHT trial. Diabetes Care. 2018;41:2147-54.

23. Yki-Järvinen $\mathrm{H}$, Bergenstal $\mathrm{R}$, Ziemen $\mathrm{M}$, et al. New insulin glargine 300 units/mL versus glargine 100 units/mL in people with type 2 diabetes using oral agents and basal insulin: glucose control and hypoglycemia in a 6-month randomized controlled trial (EDITION 2). Diabetes Care. 2014;37:3235-43.

24. Davies M, Bain S, Charpentier G, et al. A randomized controlled, treat-to-target study evaluating the efficacy and safety of insulin glargine $300 \mathrm{U} / \mathrm{mL}$ (Gla-300) administered using either device-supported or routine titration in people with type 2 diabetes. J Diabetes Sci Technol. 2019. https://doi. org/10.1177/1932296818821706.

25. Philis-Tsimikas A, Brod M, Niemeyer M, Ocampo Francisco AM, Rothman J. Insulin degludec oncedaily in type 2 diabetes: simple or step-wise titration (BEGIN: once simple use). Adv Ther. 2013;30:607-22.

26. Strojek K, Bigot G, Bonnemaire M, et al. Self- vs. physician-led titration of insulin glargine $300 \mathrm{U} / \mathrm{mL}$ (Gla-300)_improved or comparable efficacy at week 24 without increased risk of hypoglycemia, irrespective of age $(<65$ or $=65$ years $)$-TAKE CONTROL. Diabetes. 2018;67(Suppl 1):A81.

27. Becker RH, Dahmen R, Bergmann K, Lehmann A, Jax T, Heise T. New insulin glargine 300 units $/ \mathrm{mL}$ provides a more even activity profile and prolonged glycemic control at steady state compared with insulin glargine 100 units/mL. Diabetes Care. 2015;38:637-43.

28. Bergenstal RM, Bailey TS, Rodbard D, et al. Comparison of insulin glargine 300 units $/ \mathrm{mL}$ and 100 units/mL in adults with type 1 diabetes: continuous glucose monitoring profiles and variability using morning or evening injections. Diabetes Care. 2017;40:554-60.

29. Home PD, Bergenstal RM, Bolli GB, et al. New insulin glargine 300 units/mL versus glargine 100 units/mL in people with type 1 diabetes: a randomized, phase 3a, open-label clinical trial (EDITION 4). Diabetes Care. 2015;38:2217-25. 
30. Ritzel R, Roussel R, Bolli GB, et al. Patient-level meta-analysis of the EDITION 1, 2 and 3 studies: glycaemic control and hypoglycaemia with new insulin glargine $300 \mathrm{U} / \mathrm{mL}$ versus glargine 100 $\mathrm{U} / \mathrm{mL}$ in people with type 2 diabetes. Diabetes Obes Metab. 2015;17:859-67.

31. Yki-Järvinen $\mathrm{H}$, Bergenstal RM, Bolli $\mathrm{GB}$, et al. Glycaemic control and hypoglycaemia with new insulin glargine $300 \mathrm{U} / \mathrm{ml}$ versus insulin glargine $100 \mathrm{U} / \mathrm{ml}$ in people with type 2 diabetes using basal insulin and oral antihyperglycaemic drugs: the EDITION 2 randomized 12-month trial including 6-month extension. Diabetes Obes Metab. 2015;17:1142-9.

32. Matsuhisa M, Koyama M, Cheng X, et al. Sustained glycaemic control and less nocturnal hypoglycaemia with insulin glargine 300U/mL compared with glargine $100 \mathrm{U} / \mathrm{mL}$ in Japanese adults with type 1 diabetes (EDITION JP 1 randomised 12-month trial including 6-month extension). Diabetes Res Clin Pract. 2016;122:133-40.

33. Heise T, Hövelmann U, Nosek L, Hermanski L, Bøttcher SG, Haahr H. Comparison of the pharmacokinetic and pharmacodynamic profiles of insulin degludec and insulin glargine. Expert Opin Drug Metab Toxicol. 2015;11:1193-201.

34. Rodbard HW, Gough S, Lane W, Korsholm L, Bretler DM, Handelsman Y. Reduced risk of hypoglycemia with insulin degludec versus insulin glargine in patients with type 2 diabetes requiring high doses of basal insulin: a meta-analysis of 5 randomized begin trials. Endocr Pract. 2014;20:285-92.

35. Wysham C, Bhargava A, Chaykin L, et al. Effect of insulin degludec vs insulin glargine U100 on hypoglycemia in patients with type 2 diabetes: the SWITCH 2 randomized clinical trial. JAMA. 2017;318:45-56.

36. Lane W, Bailey TS, Gerety G, et al. Effect of insulin degludec vs insulin glargine U100 on hypoglycemia in patients with type 1 diabetes: the SWITCH 1 randomized clinical trial. JAMA. 2017;318:33-44.

37. Marso SP, McGuire DK, Zinman B, et al. Efficacy and safety of degludec versus glargine in type 2 diabetes. N Engl J Med. 2017;377:723-32.

38. Landstedt-Hallin L. Changes in HbA1c, insulin dose and incidence of hypoglycemia in patients with type 1 diabetes after switching to insulin degludec in an outpatient setting: an observational study. Curr Med Res Opin. 2015;31:1487-93.

39. Zhou FL, Ye F, Gupta V, et al. Lower risk of hypoglycemia after switch to insulin glargine $300 \mathrm{U} / \mathrm{mL}$ (Gla-300) vs other basal insulins in patients with type 2 diabetes (T2D) on basal insulin in real-world clinical settings (Deliver 2 study). Poster presented at the Endocrine Society 2017 Annual Meeting (ENDO 2017), Orlando, FL, USA; April 1-4, 2017; poster LB SUN 81.

40. Zhou FL, Ye F, Gupta V, et al. Older adults with type 2 diabetes (T2D) experience less hypoglycemia when switching to insulin glargine $300 \mathrm{U} / \mathrm{mL}$ (Gla300) vs other basal insulins (DELIVER 3 Study). Poster presented at the American Diabetes Association (ADA) 77th Scientific Sessions, San Diego, CA, USA; June 10, 2017; poster 986-P.

41. Bailey TS, Zhou FL, Gupta R, et al. Glycemic goal attainment and hypoglycemia risk outcomes in patients with T2D intiating insulin glargine 300 $\mathrm{U} / \mathrm{mL}$ vs $100 \mathrm{U} / \mathrm{mL}$ in real-world clinical practice. Poster presented at the 2018 Annual Meeting of the Academy of Managed Care Pharmacy (AMCP), Boston, MA, USA; April 23-26, 2018; poster E13.

42. Sullivan SD, Bailey TS, Roussel R, et al. Clinical outcomes in real-world patients with type 2 diabetes switching from first- to second-generation basal insulin analogues: comparative effectiveness of insulin glargine 300 units $/ \mathrm{mL}$ and insulin degludec in the DELIVER D+ cohort study. Diabetes Obes Metab. 2018;20:2148-58.

43. Bohn B, Bramlage $\mathrm{P}$, Wagner $\mathrm{C}$, et al. Which patients from routine care use the new insulin analogue glargine U300 compared to patients with glargine U100: a multicenter analysis of 14,123 patients with insulin glargine from die diabetes registries DPV and DIVE. Wien Med Wochenschr. 2018;168:415-22.

44. Meneghini L, Sullivan S, Oster G, et al. A randomized prospective pragmatic real-world clinical trial of insulin glargine $300 \mathrm{u} / \mathrm{ml}$ versus other basal insulins in insulin-naive patients with type 2 diabetes: a 6-month analysis of the achieve control study. J Manag Care Pharm. 2018;24:S18.

45. Roussel R, Ritzel R, Boëlle-Le Corfec E, Balkau B, Rosenstock J. Clinical perspectives from the BEGIN and EDITION programmes: trial-level meta-analyses outcomes with either degludec or glargine $300 \mathrm{U} / \mathrm{mL}$ versus glargine $100 \mathrm{U} / \mathrm{mL}$ in T2DM. Diabetes Metab. 2018;44:402-9.

46. Meneghini L, Zhou FL, Bosnyak Z, et al. Hypoglycemia risk associated with basal insulin use in type 2 diabetes (T2DM): the LIGHTNING study. Poster presented at the 11th Annual Conference on Advanced Technologies and Treatments for Diabetes (ATTD), Vienna, Austria; February 1, 2018; poster ATTD8-0420.

47. Tibaldi JM, Haldrup S, Sandberg V, Wolden ML, Rodbard HW. Clinical outcome assessment of the 
effectiveness of insulin degludec (degludec) in reallife medical practice (CONFIRM) - a comparative effectiveness study of degludec and insulin glargine $300 \mathrm{U} / \mathrm{mL}$ (Glargine U300) in insuline-naïve patients with type 2 diabetes (T2D). Diabetes. 2018;67(Suppl 1):LB27.

48. Sutton G, Minguet J, Ferrero C, Bramlage P. U300, a novel long-acting insulin formulation. Expert Opin Biol Ther. 2014;14:1849-60.
49. Steinstraesser A, Schmidt R, Bergmann K, Dahmen R, Becker RH. Investigational new insulin glargine 300 $\mathrm{U} / \mathrm{ml}$ has the same metabolism as insulin glargine 100 U/ml. Diabetes Obes Metab. 2014;16:873-6.

50. Jonassen I, Havelund S, Hoeg-Jensen T, Steensgaard DB, Wahlund PO, Ribel U. Design of the novel protraction mechanism of insulin degludec, an ultra-long-acting basal insulin. Pharm Res. 2012;29:2104-14. 